\title{
Physiological variables during open chest cardiopulmonary resuscitation: results from a small series
}

\author{
J Calinas-Correia, I Phair
}

\begin{abstract}
Objectives-To evaluate the efficacy of open chest cardiac compressions for the resuscitation of pre-hospital cardiac arrest patients presenting with nonshockable rhythms on arrival at the emergency department.

Design-Prospective observational study of outcomes and physiological parameters during open chest cardiac compressions for non-traumatic pre-hospital cardiac arrest.
\end{abstract}

Setting-Large accident and emergency diac arrest patients with pre-hospital advanced life support provided by ambulance service paramedics.

Subjects-All patients in whom open chest cardiac compressions were performed by the author (JCC) during the period from January to May 1998 (seven patients).

Interventions-Assessment of artificial pulse and blood pressure generated by open chest cardiac compressions, measurement of arterial blood gases, recovery of spontaneous cardiac output, recovery of spontaneous ventilatory efforts and survival to admission and to discharge.

Results-Artificial pulse and recordable non-invasive blood pressures were generated in all patients; $\mathrm{Po}_{2}$ was physiological or supra-physiological in all patients; $\mathbf{P C O}_{2}$ was physiological or subphysiological in all patients; $\mathrm{pH}$ and base deficit were not corrected in the five patients with repeated samples (including two receiving $50 \mathrm{mEq}$ sodium bicarbonate); three patients recovered spontaneous cardiac output (ROSC); two patients recovered spontaneous respiratory efforts (unrelated to ROSC); no patients survived to admission.

Conclusions-Open chest cardiac compressions provide effective perfusion, enabling correction of ventilation parameters and showing clinical signs of adequate perfusion. However, acidosis was not corrected and the use of $50 \mathrm{mEq}$ sodium bicarbonate was ineffective.

(F Accid Emerg Med 2000;17:201-204)

Keywords: thoracotomy; open chest cardiac compressions; pre-hospital cardiac arrest; advanced life support

Dr Calinas-Correia

16 Roskear, Camborne,

Cornwall TR14 8DN

(e-mail:

designworks@fsbdial.co.uk)

Accepted for publication 25 October 1999 The efficacy of open chest cardiac compressions (OCCC) to provide adequate systemic and cerebral perfusion is well established in the department receiving pre-hospital car-

medical literature. ${ }^{1-10}$ The work of Rubertsson et $a l^{11}$ in an animal model, demonstrated the better systemic perfusion achieved with OCCC, especially during the important relaxation phase when coronary perfusion occurs. On human cardiac arrest, Boczar et $a l^{2}$ found a significant difference between the coronary perfusion pressure achieved with closed chest $(7.3+/-5.7 \mathrm{~mm} \mathrm{Hg})$ and open chest $(32.6+/-$ $17.8 \mathrm{~mm} \mathrm{Hg}$ ) cardiac compressions. Open chest technique was the only one to provide more than $15 \mathrm{~mm} \mathrm{Hg}$, the level recommended to achieve return of spontaneous cardiac output (ROSC). ${ }^{3}$ Cerebral resuscitability seems to be increased by the absence of increased intracranial pressure associated with increased intrathoracic pressure on the compression phase of chest compressions, ${ }^{6-8}$ and post-resuscitation physiological parameters are also better with open chest techniques. ${ }^{89}$

Resuscitability decreases over time despite optimal management. Safar ${ }^{12}$ recognised unresponsiveness to closed chest compressions as an indication for thoracotomy. Closed chest compressions have dubious benefits in prolonged asystole or electromechanical dissociation resistant to advanced life support (ALS), discontinuation of resuscitation being advised after 15 minutes. ${ }^{13}$ Open chest techniques could offer the opportunity to improve the outcome in those patients in asystole or electromechanical dissociation who do not respond to conventional ALS within this period of time.

The frequent use of the open chest technique in the department led to the design of a data collection form to evaluate the efficacy of the technique. Our aims were to assess the effect of OCCC on physiological variables and outcomes in adult patients suffering prehospital non-traumatic cardiac arrest, presenting with non-shockable rhythms on arrival at the emergency department.

\section{Methods}

DESIGN

Prospective observational study of outcomes and physiological parameters during OCCC for non-traumatic pre-hospital cardiac arrest.

\section{SETTING}

The North Staffordshire Hospital emergency department has a designated resuscitation team, led by the accident and emergency registrar or the critical care fellow. The department receives the pre-hospital cardiac arrests for the North Staffordshire area. The pre-hospital ALS interventions are delivered by the Staf- 
SUBJECTS

The criteria for inclusion were: cardiac arrest with a non-shockable rhythm on arrival at the department, failure to to achieve ROSC with adequate pre-hospital ALS (with closed chest compressions, patent tracheal tube and ventilated, and patent intravenous access), and failure to achieve ROSC during the first minutes of inhospital treatment. Low output in patients with electromechanical dissociation was excluded with Doppler, as described elsewhere. ${ }^{14}$

The final decision to perform OCCC was taken by the resuscitation team leader, taking into account the length or absence of basic life support, the length of ALS, the absence of obvious causes for the condition (for example, pneumothorax, hypovolaemia, hypothermia, etc), the absence of indications for other interventions (for example, pacing for maintained atrial activity) and the absence of obvious contraindications for resuscitation.

This report concerns the interventions performed from January to May 1998. Individual arrest data were collected following the out of hospital arrest Utstein Style recommendations, ${ }^{15}$ as applicable to individual patients.

INTERVENTIONS

Except for the thoracotomy and OCCC, ALS protocols ${ }^{1316}$ were followed.
Thoracotomy was performed through a scalpel incision over the left 5 th rib from sternum to mid-axillary line and blunt digital dissection of the 4th intercostal space muscles. A rib spreader was introduced, and the heart compressed manually approximately 100/min. Time from stopping of chest compressions to start of direct cardiac compressions was less than one minute. Efficacy was surveyed by femoral pulse palpation and non-invasive blood pressure monitoring.

\section{OUTCOMES}

Measured physiological variables were: artificial pulse palpation, non-invasive blood pressure (automated measurements), arterial blood gases, recovery of spontaneous cardiac output, recovery of spontaneous respiratory efforts and survival to admission and to discharge.

\section{Results (table 1)}

From January to May 1998, thoracotomies for resuscitation were performed in seven patients. Ages ranged from 49 to 74 years (mean 63), all being white men. Five were witnessed arrests, and four had bystander basic life support (BLS) of variable quality, for an average of six minutes. ALS was administered to all patients by Staffordshire Ambulance Service Paramedics after an average delay of 7.3 minutes, for an average of 30.3 minutes with an average

Table 1 Results

\begin{tabular}{|c|c|c|c|c|c|c|c|}
\hline Patient number & 1 & 2 & 3 & 4 & 5 & 6 & 7 \\
\hline Sex & M & $\mathrm{M}$ & $\mathrm{M}$ & $\mathrm{M}$ & $\mathrm{M}$ & M & $\mathrm{M}$ \\
\hline Age & 60 & 71 & 63 & 65 & 49 & 58 & 73 \\
\hline Witnessed & yes & yes & no & no & yes & yes & yes \\
\hline Time to initiation of life support (basic or advanced) & 0 & 0 & ? & $7 ?$ & 7 & 1 & 6 \\
\hline BLS duration (min) & 13 & 4 & 4 & 0 & 0 & 9 & 0 \\
\hline First rhythm & $\mathrm{vf}$ & vf & asyst & asyst & vf & asyst & asyst \\
\hline ALS time ( $\min )$ & 18 & 31 & 39 & 28 & 19 & 53 & 24 \\
\hline Pre-hospital time ( $\mathrm{min})$ & 31 & 35 & 43 & 35 & 26 & 63 & 30 \\
\hline Rhythm on arrival & asyst & emd & emd & asyst & asyst & asyst & asyst \\
\hline Time from arrival to thoracotomy ( $\mathrm{min})$ & 13 & 5 & 5 & 5 & 3 & 3 & 5 \\
\hline Total time to thoracotomy & 44 & 40 & $48 ?$ & $40 ?$ & 29 & 66 & 35 \\
\hline Asystole time (min) & 30 & $\mathrm{n} / \mathrm{a}$ & 1 & 33 & 21 & 56 & 29 \\
\hline \multicolumn{8}{|c|}{$\begin{array}{l}\text { ?, Uncertainty because of unwitnessed arrest; n/a, not applicable; BLS, Basic Life Support; ALS, Advanced Life Support; } \\
\text { VF, ventricular fibrillation; EMD, electromechanical dissociation; Asyst, asystole. }\end{array}$} \\
\hline \multicolumn{8}{|l|}{ B Outcomes } \\
\hline Spontaneous breathing & no & yes & no & no & no & yes & no \\
\hline ROSC achieved & yes & no & no & yes & no & no & yes \\
\hline ROSC duration (min) & 3 & 0 & 0 & $19+9+5$ & 0 & 0 & 5 \\
\hline Time from thoracotomy to ROSC ( $\mathrm{min}$ ) & 2 & & & 3 & & & 8 \\
\hline Survival & \multicolumn{7}{|c|}{ no patient survived to admission } \\
\hline Total arrest duration (min) & 51 & 60 & 70 & 86 & 61 & 95 & 75 \\
\hline \multicolumn{8}{|l|}{ ROSC, return of spontaneous cardiac output. } \\
\hline \multicolumn{8}{|l|}{$C$ Post-thoracotomy data (during OCCC) } \\
\hline Patient number & 1 & 2 & 3 & 4 & 5 & 6 & 7 \\
\hline \multicolumn{8}{|c|}{ Blood pressure - automated measurements (NIBP) (mm Hg) } \\
\hline Systolic_-1st measurement & 156 & 150 & 60 & 88 & 154 & 138 & 96 \\
\hline Diastolic-1 st measurement & 112 & 100 & 30 & 59 & 117 & 100 & 53 \\
\hline Systolic -2 nd measurement & * & 136 & - & * & 87 & 159 & $\star$ \\
\hline Diastolic-2nd measurement & * & 100 & - & $\star$ & 53 & ? & $\star$ \\
\hline \multicolumn{8}{|c|}{$\begin{array}{l}\text { ^No further NIBP measurements presented because of ROSC. } \\
\text { Acidosis evolution }\end{array}$} \\
\hline $\mathrm{pH}-1$ st sample & 7.11 & 7.175 & 6.967 & 6.707 & 7.021 & 6.954 & 7.174 \\
\hline Bicarb $(\mathrm{mEq})$ & & & & 50 & 50 & 50 & \\
\hline Interval to 2 nd sample ( $\mathrm{min}$ ) & & 15 & 8 & 23 & & 7 & 8 \\
\hline $\mathrm{pH}-2$ nd sample & - & 7.091 & 6.938 & $6.689^{\star}$ & - & 6.879 & 7.322 \\
\hline BE-1st sample & -20.8 & -18.9 & -18.6 & -27.0 & -16.8 & -12.7 & -7.2 \\
\hline $\mathrm{BE}-2$ nd sample & & -21.6 & -21.5 & $-27.3^{\star}$ & & -24.3 & -9.9 \\
\hline \multicolumn{8}{|l|}{ Arterial blood gases analysis } \\
\hline $\mathrm{PO}_{2}-1$ st sample & 36.84 & 31.61 & 7.86 & 14.60 & 13.47 & 2.54 & 68.96 \\
\hline $\mathrm{Po}_{2}-2$ nd sample & & 51.26 & 10.48 & $41.55^{\star}$ & & 49.33 & 69.51 \\
\hline $\mathrm{PCO}_{2}-1$ st sample & 3.05 & 3.02 & 6.72 & 5.50 & 7.70 & 11.21 & 7.52 \\
\hline $\mathrm{PCO}_{2}-2$ nd sample & & 2.92 & 5.40 & $5.64^{\star}$ & & 4.54 & 3.98 \\
\hline
\end{tabular}


pre-hospital time of 37.6 minutes. On arrival to the emergency department, all patients had the trachea intubated and intravenous access established.

Presentation rhythm to the ambulance crew was asystole in four patients and ventricular fibrillation in the other three. On arrival at the emergency department, five patients were in asystole and two in electromechanical dissociation (one wide complex bradycardia and the other sinus rhythm).

Thoracotomy was performed within five minutes of arrival in all but one patient.

With manual compressions of the heart, all patients had palpable femoral pulses and recordable non-invasive blood pressure.

There was great variation of first recorded $\mathrm{PO}_{2}$ and $\mathrm{PCO}_{2}$ values between patients, but of those five patients with repeated samples, only one remained below $40 \mathrm{kPa}$ and all had acceptable $\mathrm{PCO}_{2}$ readings. On the other hand, $\mathrm{pH}$ and base deficit worsened in all but one patient despite the other indicators of adequate ventilation and perfusion, and, in two patients despite $50 \mathrm{mEq}$ sodium bicarbonate, administered immediately after initial assessment of arterial blood gases.

Three patients had periods of spontaneous cardiac output shortly after initiation of OCCC. Two patients recovered spontaneous respiratory efforts while on open chest cardiac massage for as long as cardiac compressions were maintained; there was no spontaneous electrical or mechanical cardiac activity and these two patients never had any period of spontaneous cardiac output.

OCCC was maintained for an average of 28 minutes, ranging from 7 to 46 minutes. For the three patients achieving ROSC, one had OCCC for seven minutes, and the other two for 40 and 46 minutes. Regarding the patients achieving spontaneous breathing, OCCC was performed for 20 and 29 minutes. Those who did not achieve spontaneous breathing or ROSC had OCCC for 22 and 32 minutes.

No patient survived to admission.

\section{Discussion}

In this series, pre-hospital times were uniformly around 35 minutes, with ALS time around 25 minutes. This seems excessive, and

Table 2 Indicators of poor outcome

\begin{tabular}{llllllll}
\hline Patient & 1 & 2 & 3 & 4 & 5 & 6 & 7 \\
Unwitnessed arrest & & & yes & yes & & & \\
Time without life support $>5$ minutes & & & & yes & yes & & yes \\
Asystole $>15$ minutes (with ALS) & yes & & & yes & yes & yes & yes \\
No ROSC after ALS $>30$ minutes & yes & yes & yes & yes & & yes & \\
Total arrest time $\geqslant 40$ minutes & yes & yes & yes & yes & yes & yes & \\
\hline
\end{tabular}

ALS and total times measured to thoracotomy.

Table 3 Factors influencing decision to pursue resuscitation

\begin{tabular}{|c|c|c|c|c|c|c|c|}
\hline Patient & 1 & 2 & 3 & 4 & 5 & 6 & 7 \\
\hline Age $\leqslant 65$ & yes & & yes & yes & yes & yes & \\
\hline Immediate life support & yes & yes & & & & yes & \\
\hline Time to ALS $<5 \mathrm{~min}$ & & yes & & & & & \\
\hline Presentation in VF & yes & yes & & & yes & & \\
\hline Change in rhythm (asystole to EMD) & & & yes & & & & \\
\hline Previous ROSC & & & & & & & yes* \\
\hline
\end{tabular}

^Pre-hospital, then asystole again before arrival. we would recommend an effort to shorten the ALS intervention time.

All patients included in this series had several indicators of poor outcome (table 2). In all patients, a decision to stop resuscitation efforts could have been justified. In each one of them the team felt further efforts were warranted (table 3), and the team leader decided to continue resuscitation.

When a decision to continue resuscitation efforts was taken, and the patients complied with the criteria presented, OCCC was performed, the rationale for decision being the failure of closed chest techniques to achieve any benefits during a length of time accepted in current recommendations. ${ }^{13}$

The timing to thoracotomy was in accordance with Takino and Okada ${ }^{4}$ less than five minutes from arrival at the emergency department (in all but one patient).

All patients had palpable femoral pulses during OCCC. It is important to note that the concomitant assessment of efficacy by femoral pulse palpation and generation of measurable automated non-invasive blood pressure made apparent the need to increase the energy of OCCC in several instances.

Despite the correction of $\mathrm{PO}_{2}$ and $\mathrm{PCO}_{2}$, acidosis worsened in all but one patient. Fifty $\mathrm{mEq}$ of sodium bicarbonate were used in three patients and shown to be ineffective in two who had repeated arterial blood sampling. Interestingly, the one who showed an improvement had not received sodium bicarbonate. These results seem to challenge the assumption that correct ventilation is the method of choice to correct $\mathrm{pH}$, and suggest the need for a better understanding of the course of profound acidosis during prolonged arrest.

During two years (1996 to 1998), the Staffordshire Ambulance Service attended 3025 cardiac arrests, ROSC being achieved in 283 , with 64 survivors. ${ }^{17}$ This yields a rate of $9.4 \%$ for ROSC and $2.1 \%$ for survival.

Regarding the outcomes evaluated in our series, three patients achieved ROSC, two resumed spontaneous breathing (unrelated to ROSC), and no patient survived to admission. Of those patients who achieved ROSC, it happened two, three or eight minutes from thoracotomy. The early achievement of ROSC after initiation of OCCC is in accordance with the expected improvement on coronary and systemic perfusion. The absence of survivors in such a small series of out of hospital cardiac arrests is expected, both from the sample size and from the selection bias-non-shockable rhythms and poor outcome indicators.

The achievement of near physiological values for non-invasive blood pressure, $\mathrm{PO}_{2}$ and $\mathrm{PCO}_{2}$, with two in seven patients recovering spontaneous respiratory efforts on the sole basis of perfusion achieved by OCCC-they had no electrical or mechanical cardiac activity - and three achieving ROSC after a brief period of OCCC, seems to demonstrate a very good efficacy of this method to achieve coronary, lung and brain stem perfusion.

Ryan et $a l^{18}$ showed that cuff blood pressure may not always be related to cardiac output. 
Here, the improvement in arterial blood gases showed pulmonary perfusion, and we believe a physiological value for non-invasive blood pressure, along with other indicators of systemic perfusion, may be understood as minimally adequate perfusion, even without a direct relation to artificial cardiac output. Arterial lines were not practical, as only the team leader was trained to insert them, and only patient 4 had ROSC of sufficient duration for it to be considered.

Safar ${ }^{12}$ recommended OCCC in suspected massive pulmonary embolism, profound hypothermia, failure to produce an artificial arterial pulse with chest compressions and prolonged arrest times with failure of chest compressions. Current recommendations for OCCC include hyperinflation of the lungs, ${ }^{16}$ suspected pericardial tamponade or ineffectiveness of closed chest compressions because of anatomical constraints. ${ }^{13}$ Despite the body of evidence in favour of better performance of open chest techniques, ${ }^{1-10}$ teaching and performance of OCCC is still exceptional in those situations, preventing a full evaluation of the impact on outcomes. Baskett's recommendation to discontinue resuscitation after 15 minutes of asystole unresponsive to conventional $\mathrm{ALS}^{13}$ is not correlated with Safar's recommendation of OCCC in prolonged arrest times ${ }^{12}$ - this indication is not listed in current resuscitation guidelines. ${ }^{1316}$ We believe open chest techniques may improve outcome in this group of patients. The predicted poor outcome with closed chest compressions, and the theoretical advantages of open chest cardiac compressions collaborate to provide the rationale for benefit and the ethical acceptability of large prospective trials.

Our study considered the practice already in place. The absence of controls receiving closed chest compressions is a consequence of this, and also of the small numbers involved. However, this will have to be investigated if the risk/benefit of OCCC compared with closed chest compressions is to be established in larger prospective trials, but will need large numbers to overcome the variability of time intervals between pre-hospital interventions, which significantly affect survivability.

The Hachimi-Idrissi series ${ }^{5}$ of 33 prehospital thoracotomies shows the feasibility of open chest resuscitation, even in that challenging environment. The BLS and ALS times presented are not dissimilar to ours, and, with the caution due to the small size of our sample, ROSC achievement is also similar.

The work of Buckman et $a l^{1920}$ on minimally invasive direct cardiac massage is likely to make this technique less invasive, while retaining its efficacy.

\section{Conclusions}

The improvement in $\mathrm{PO}_{2}$ and $\mathrm{PCO}_{2}$ observed in this small series suggests effective pulmonary perfusion, and the recovery of spontaneous respiratory efforts in two patients during OCCC is strongly suggestive of effective brain stem perfusion. The short time from thoracotomy to achievement of ROSC in three patients suggests improved coronary perfusion. Together with the generation of near physiological automated blood pressure measurements, our results suggest adequate systemic perfusion with OCCC.

The failure of effective perfusion and ventilation to control acidosis, and the failure of 50 $\mathrm{mEq}$ of sodium bicarbonate to produce visible effects, deserve further investigation.

The results of this small series support the usefulness of larger prospective trials.

Contributor

João Calinas-Correia was the resuscitation team leader involved with all the patients included in the study, and was responsible for the data collection and writing of the paper. Ivan Phair discussed core ideas and participated in the interpretation of the results and the writing of the paper.

Funding: none.

Conflicts of interest: none.

1 Rubertsson S, Grenvik A, Wiklund L. Blood flow and perfusion pressure during open-chest versus closed-chest cardiopulmonary resuscitation in pigs. Crit Care Med 1995;23:715-25.

2 Boczar ME, Howard MA, Rivers EP, et al. A technique revisited: hemodynamic comparison of closed and openchest cardiac massage during human cardiopulmonary chest cardiac massage during human cardiopu

3 Paradis NA, Martin GB, Rivers EP, et al. Coronary perfusion pressure and the return of spontaneous circulation in human cardiopulmonary resuscitation. $f A M A$ 1990;263:1106-13.

4 Takino M, Okada Y. The optimum timing of resuscitative thoracotomy for non-traumatic out-of-hospital cardiac arrest. Resuscitation 1993;26:69-74.

5 Hachimi-Idrissi S, Leeman J, Hubloue Y, et al. Open chest cardio-pulmonary resuscitation in out-of-hospital arrest. Resuscitation 1997;35:151-6.

6 Bircher NG, Safar P, Stewart R. A comparison of standard MAST-augmented and open-chest CPR in dogs. Crit Care Med 1980;8:147-52.

7 Bircher NG, Safar P. Comparison of standard and "new" closed-chest CPR and open-chest CPR in dogs. Crit Care Med 1981;9:384-5.

8 Alifimoff JK, Safar P, Bircher NG. Opening the chest to keep the brain alive in prolonged cardiopulmonary resuscitation. Prehospital and Disaster Medicine 1985;1:233-6.

9 Bircher NG, Safar P. Cerebral preservation during cardiopulmonary resuscitation. Crit Care Med 1985;13:185-90.

10 Tisherman SA, Vandevelde K, Safar P. Future directions for resuscitation research-V. Resuscitation 1997;34:281-93.

11 Rubertsson S, Grenvik A, Zemgulis V, et al. Systemic perfusion pressure and blood flow before and after administration of epinephrine during experimental cardiopulistration of epinephrine during experimental cardiop

12 Safar P, Bircher NG. Cardiopulmonary cerebral resuscitation. 3rd ed. London: WB Saunders, 1988:212-19.

13 European Resuscitation Council. Guidelines for resuscitation. Antwerp: European Resuscitation Council, 1996.

14 Calinas-Correia J, Phair I. Is there a pulse?. Resuscitation 1999;41:201-2.

15 Cummins RO, Chamberlain DA, Abramson NS, et al. Recommended guidelines for uniform reporting of data from out-of-hospital cardiac arrest: The Utstein Style. Ann out-of-hospital cardiac arres:
Emerg Med 1991;20:861-74.

16 Resuscitation Council (UK). Advanced Life Support Courseprovider manual. 3rd ed. London: Resuscitation Council (UK), 1998.

17 Devlin P. Audit of pre-hospital cardiac arrest April 1997 to March 1998. Stafford: Staffordshire Ambulance Service, August 1998

18 Ryan BP, Redmond $\mathrm{AD}$, Edwards JD. When to stop resuscitation: the significance of cuff blood pressure. Arch Emerg Med 1991;8:177-81.

19 Buckman RFJr, Badellino MM, Mauro LH, et al. Direct cardiac massage without major thoracotomy: feasibility and systemic blood flow. Resuscitation 1995;29:237-48.

20 Buckman RFJr, Badellino MM, Eynon CA, et al. Openchest cardiac massage without major thoracotomy: metabolic indicators of coronary and cerebral perfusion. Resuscitation 1997;34:247-53. 